\title{
Erratum to: Four new Penicillium species isolated from the fynbos biome in South Africa, including a multigene phylogeny of section Lanata-Divaricata
}

\author{
Cobus M. Visagie ${ }^{1,2,3}$. Jos Houbraken ${ }^{3}$ - Keith A. Seifert ${ }^{2}$ - Robert A. Samson ${ }^{3}$. \\ Karin Jacobs ${ }^{1}$
}

Published online: 28 November 2015

(C) German Mycological Society and Springer-Verlag Berlin Heidelberg 2015

\section{Erratum to: Mycol Progress}

DOI: $10.1007 / \mathbf{s} 11557-015-1118-Z$

The original version of this article unfortunately contained a mistake. In the Penicillium malacosphaerula description text stated:

“Holotypus — South Africa, Malmesbury, (3329'46”S $18^{\circ} 35^{\prime} 16^{\prime} \mathrm{E}$ ), from soil, 21 February 2007 , collected and isolated by CM Visagie, CBS H-21332 (ex-typus: CBS 135121 $=\mathrm{CV} 2855=$ DTO 180-E6 = DAOM 241161).

Additional cultures examined - South Africa, Malmesbury, (3329'46”S 18³5'16”E), from soil, 21 February 2007, collected and isolated by CM Visagie, CBS $\mathbf{1 3 5 1 2 0}=$ CV $2836=$ DTO 180-D 1, CBS $135122=$ CV 2848 = DTO 180-E1."

The bold text should be switched around to read:

"Holotypus - South Africa, Malmesbury, (3329'46"S 18³5'16"E), from soil, 21 February 2007, collected and isolated by CM Visagie, CBS H-21332 (ex-typus: CBS 135120 = CV 2855 = DTO 180-E6 = DAOM 241161).

Additional cultures examined - South Africa, Malmesbury, (3329'46"S 18 35'16”E), from soil, 21 February 2007, collected and isolated by CM Visagie, CBS $135121=$ CV $2836=$ DTO 180-D1, CBS 135122 = CV $2848=$ DTO 180-E1."

The online version of the original article can be found at http://dx.doi.org/ 10.1007/s11557-015-1118-z.

Karin Jacobs

kj@sun.ac.za

Cobus M. Visagie

cobusvisagie9@gmail.com

Jos Houbraken

j.houbraken@cbs.knaw.nl

Keith A. Seifert

keith.seifert@agr.gc.ca

Robert A. Samson

r.samson@cbs.knaw.nl

1 Department of Microbiology, University of Stellenbosch, Private

Bag X1, Stellenbosch 7600, South Africa

2 Biodiversity (Mycology), Agriculture and Agri-Food Canada, Ottawa, ON, Canada K1A 0C6

3 CBS-KNAW Fungal Biodiversity Centre, Uppsalalaan 8, 3584 CT Utrecht, The Netherlands 